\title{
Dietary supplementation with astaxanthin may ameliorate sperm parameters and DNA integrity in streptozotocin-induced diabetic rats
}

\author{
Maryam Bahmanzadeh ${ }^{1}$, Aliasghar Vahidinia ${ }^{2,3}$, Shayesteh Mehdinejadiani ${ }^{4}$, Saeed Shokri ${ }^{5}$, Zohreh Alizadeh ${ }^{1}$ \\ ${ }^{1}$ Endometrium and Endometriosis Research Center, Hamadan University of Medical Sciences, Hamadan; ${ }^{2}$ Nutrition Health research center, Hamadan \\ University of Medical Sciences, Hamadan; ${ }^{3}$ Department of Nutrition, School of Medicine, Hamadan University of Medical Sciences, Hamadan; \\ ${ }^{4}$ Department of Anatomy, School of Medicine, Tehran University of Medical Sciences, Tehran; ${ }^{5}$ Department of Anatomical Sciences, School of Medicine, \\ Zanjan University of Medical Sciences, Zanjan, Iran
}

Objective: Diabetes mellitus (DM) is known to cause many systemic complications as well as male infertility. Astaxanthin (ASTX) is a powerful antioxidant that is involved in a variety of biologically active processes, including those with anti-diabetes effects. The present study investigates the effect of ASTX on the spermatozoa function in streptozotocin (STZ)-induced diabetic rats.

Methods: We divided 30 adult rats into three groups (10 rats per group), with a control group that received corn oil mixed with chow. DM was induced by intra-peritoneal injection of STZ. Eight weeks after the STZ injection, half of the diabetic animals were used as diabetic controls, and the rest were treated with ASTX for 56 days. Then the parameters and chromatin integrity of the epididymal sperm were analyzed using chromomycin $A 3$, toluidine blue (TB), and acridine orange (AO) staining.

Results: The count, viability, and motility of the epididymal sperm were decreased significantly in the STZ group in comparison with the control group (count and viability, $p<0.001$; motility, $p<0.01$ ). ASTX increased normal morphology and viable spermatozoa compared to the STZ group (morphology, $p=0.001$; viability, $p<0.05$ ). The percentage of abnormal chromatins in TB and AO staining was higher in the STZ group compared to the control group $(p<0.001)$. The mean percentage of TB and AO positive spermatozoa in STZ rats was significantly lower in the $\mathrm{STZ}+\mathrm{ASTX}$ group $(\mathrm{TB}, p=0.001 ; \mathrm{AO}, p<0.05)$.

Conclusion: This study observed that in vivo ASTX treatment partially attenuates some detrimental effect of diabetes. Conversely, ASTX improved sperm viability, normal morphology, and DNA integrity.

Keywords: Astaxanthin; Chromatin; Diabetes mellitus; Sperm; Streptozotocin

\section{Introduction}

Diabetes mellitus (DM) is a chronic disease, the prevalence of which

Received: Jan 5, 2016 · Revised: Mar 21, 2016 · Accepted: Apr 14, 2016 Corresponding author: Zohreh Alizadeh

Endometrium and Endometriosis Research Center, Hamadan University of Medical Sciences, Pasdaran Street, Hamadan, Iran

Tel: +98-81-38283939 Fax: +98-81-38283939 E-mail: alizadeh@umsha.ac.ir

*This research was supported by a grant from Hamadan University of Medical Sciences (no. 9403121233).

This is an Open Access article distributed under the terms of the Creative Commons Attribution Non-Commercial License (http://creativecommons.org/licenses/by-nc/4.0/) which permits unrestricted non-commercial use, distribution, and reproduction in any medium, provided the original work is properly cited. has increased rapidly worldwide. The World Health Organization (WHO) estimated that the total number of people with diabetes is anticipated to progress to 366 million by 2030 [1,2]. DM is known to cause many systemic complications and male infertility [3]. DM causes molecular alterations that negatively affect sperm quality and function [4]. Moreover, it can disrupt endocrine control of spermatogenesis [5].

Recent studies have shown that some conditions such as endocrine disorders, diabetic neuropathy, and oxidative stress may be the main causes of sperm damage [3]. Hyperglycemia, which directly induces reactive oxygen species (ROS) production and elevates the level of oxidative stress, plays a crucial role in the development of diabetes 
[6]. It has been accepted that spermatozoa are vulnerable to ROS-induced damage [7]. The oxidation of lipids, proteins, and other macromolecules such as DNA occurs during the development of diabetes [8]. Men with diabetes have been found to have a significantly higher percentage of spermatozoa with nuclear DNA damage, which subsequently alters male fertility, early embryonic growth, reproductive outcomes, and miscarriage rates [9-12].

The protective effects of exogenously administered antioxidants have been extensively studied in animal models of DM [13-15]. Antioxidants play an important role in the maintenance of sperm motility and sperm DNA integrity against oxidative damage [16]. Astaxanthin (ASTX) is a keto-carotenoid pigment extracted from the algae Haematococcus pluvialis, a species of Chlorophyta. ASTX is known to have antioxidant effects against various kinds of oxidative stress [17]. Moreover, ASTX is involved in a variety of biologically active processes, including those with anti-diabetes and anti-obesity effects [1820]. This carotenoid agent has a potential health-promoting effect in diabetic models by attenuating inflammation and apoptosis. ASTX is well known for its antioxidant properties. However, to the best of our knowledge, this is the first study to evaluate the protective role of ASTX against streptozotocin (STZ)-induced sperm damage in diabetic rats $[17,21]$.

An experimental animal model of DM is a useful method for studying the pathogenesis and treatment of this disease [22]. STZ-induced DM in rats provides a relevant model for studying the complications of diabetes [23].

ASTX, which plays a role in a variety of biological activities, has been widely studied in relation to DM and diabetic complications [24,25]. However, whether ASTX would be able to alleviate DM-induced alterations in sperm quality and DNA integrity, and its precise mechanisms, remains undetermined. Therefore, the present study was designed to investigate the impact of 2 months of ASTX treatment on the sperm parameters and DNA integrity in STZ-induced diabetic rats.

\section{Methods}

\section{Ethics}

All animal experiments were conducted in accordance with national guidelines and protocols, approved by the Institutional Animal Ethics Committee.

\section{Animals}

Thirty healthy adult male Wistar rats (200 to $250 \mathrm{~g}$ ) were kept under standard conditions. As a fertility test, all male animals were mated with adult female rats and pregnancy was evaluated (all male rats were fertile). Rats were acclimatized for 1 week, under a 12 hours light-dark cycle at $23^{\circ} \mathrm{C} \pm 2^{\circ} \mathrm{C}$. The rats were fed with standard commercial laboratory chow pellets and water ad libitum. The daily intake of animal food was monitored at least 1 week prior to the start of treatment in order to determine the amount of food needed per experimental animal. Thereafter, the rats were randomly selected and divided into three groups. Each group was comprised of 10 rats, detailed as follows: the control group received chow mixed with corn oil. The STZ group received STZ with the equivalent volume of chow mixed with corn oil. The STZ+ASTX (ASTX 10\%, Fuji Chemical Industries, Toyama, Japan) group received STZ plus an equivalent volume of chow and corn oil supplemented with ASTX $(720 \mathrm{mg} / \mathrm{kg}$ body weight) for a period of 8 weeks (the period taken to complete a spermatogenic cycle in a rat) $[26,27]$.

\section{Induction of DM}

Experimental DM was induced by a single intra-peritoneal injection of STZ (90 mg/kg, Sigma-Aldrich, St. Louis, MO, USA) in a sodium citrate buffer ( $\mathrm{pH} 4.5$ ) to overnight-fasted animals. Three days after the administration of STZ, the blood glucose level of the tail vein was measured in all animals with a portable glucose meter. Blood glucose levels of $270 \mathrm{mg} / \mathrm{dL}$ and above were considered to indicate the presence of diabetes in the model. At the end of the 8th week (56 days) after STZ injection, half of the diabetic animals were used as diabetic controls, and the rest were treated with ASTX for 56 days $[21,28]$.

\section{Sperm analysis}

Epididymal spermatozoa were collected by dissecting the caudal part of the left epididymis. The spermatozoa were separated from the epididymal tubules by chopping the caudal part of the epididymis in $5 \mathrm{~mL}$ of Ham's F-10 solution (Sigma-Aldrich). The solution was incubated for 10 minutes at $37^{\circ} \mathrm{C}$ to release the spermatozoa into the medium. After pipetting, $100 \mu \mathrm{L}$ of the sperm suspension was diluted with $900 \mu \mathrm{L}$ of saline. The diluted sperm suspension was transferred into a Neubauer hemocytometer chamber and the sperm heads were counted [29,30]; data were expressed as the total number of sperm per milliliter.

In this study, the percentage of motility was evaluated for all animals. Briefly, the spermatozoa were classified as motile or immotile. Sperm viability was also determined by using Eosin staining (SigmaAldrich) as described previously [29]. Eosin penetrated non-viable, dead spermatozoa with disrupted membranes, which stained red. The percentage of normal morphology of 100 spermatozoa per rat was assessed by light microscopy (400× Z Zeiss, Munich, Germany) as described previously [29,31]. One experienced technician blinded to the study performed all analyses. 


\section{Evaluation of sperm nuclear chromatin 1) Chromomycin $A 3$ staining}

The epididymal sperm samples were fixed in Carnoy's solution (methanol/glacial acetic acid 3:1) at $4^{\circ} \mathrm{C}$ for 5 minutes. Each slide was treated for 20 minutes with $100 \mu \mathrm{L}$ of chromomycin A3 (CMA3) solution (C2659, Sigma-Aldrich) $(0.25 \mathrm{mg} / \mathrm{mL}$ in a Mcllvain buffer: $7 \mathrm{~mL}$ citric acid $0.1 \mathrm{M}+32.9 \mathrm{~mL} \mathrm{Na} 2 \mathrm{HPO}_{4} \cdot 7 \mathrm{H}_{2} \mathrm{O} 0.2 \mathrm{M}$, pH 7.0, containing 10 $\mathrm{mM} \mathrm{MgCl} 2$ ). The slides were then rinsed in buffer and mounted with buffered glycerol (1:1). Microscopic analysis of the slides was performed on a Olympus fluorescence microscope (Zeiss), with the appropriate filters (460 to $470 \mathrm{~nm}$ ). Evaluation of CMA3 staining was carried out by distinguishing between spermatozoa with bright yellow staining (CMA3 positive) and spermatozoa with dull yellow staining (CMA3 negative), expressed as a percentage [30,32].

\section{2) Toluidine blue staining}

For toluidine blue (TB) staining, the air-dried smears of spermatozoa were fixed in fresh $96 \%$ ethanol-acetone $(1: 1)$ at $4^{\circ} \mathrm{C}$ for 30 minutes, and finally hydrolyzed in $0.1 \mathrm{~N} \mathrm{HCl}$ at $4^{\circ} \mathrm{C}$ for 5 minutes. The slides were then rinsed twice in distilled $\mathrm{H}_{2} \mathrm{O}$ for 2 minutes, and finally stained with $0.05 \%$ TB (Merck, Darmstadt, Germany) for 10 minutes. The staining buffer was composed of 50\% citrate phosphate (Mcllvain buffer, pH 3.5). Two different scores were applied: normal chromatin (light blue) and abnormal chromatin (dark blue) [32-35].

\section{3) Acridine orange staining}

Sperm DNA integrity, the smears were air-dried for 1 hour and then fixed overnight in methanol/acetic acid. Each sample was stained for 10 minutes in freshly prepared acridine orange $(\mathrm{AO} ; 0.19 \mathrm{mg} / \mathrm{mL}$, Sigma-Aldrich) in Mcllvain phosphate-citrate buffer ( $\mathrm{pH} 4)$ for 5 minutes. Smears were evaluated on the same day using a fluorescent microscope with a $460 \mathrm{~nm}$ filter. The percentage of 100 green (normal double-stranded DNA) and orange/red (abnormally denatured) fluorescence spermatozoa per sample was calculated as described previously [30,32].

\section{4) Statistical analysis}

Statistical analysis was performed by using the SPSS for Windows ver. 16.0 (SPSS Inc., Chicago, IL, USA). First, the Kruskal-Wallis test was used for the presence of statistical significance in each value. Then, one-way analysis of variance was applied for comparison between groups following Tukey's post hoc test. The $p$-values less than 0.05 were regarded as indicating significance.

\section{Results}

\section{Sperm parameters}

Table 1 shows the effect of DM and ASTX treatment on epididymal sperm characteristics. The total count of the sperm extracted from the epididymis decreased significantly $(108.75 \pm 4.8)$ in the STZ group when compared with the control group $(148.6 \pm 12.3, p<0.001)$. The mean percentage of sperm motility decreased significantly in the STZ group (43.5 \pm 5.3$)$ when compared with the control group (57.6 $\pm 5.6, p=0.004)$. In addition, the mean percentage of sperm viability decreased significantly in the STZ group $(17.5 \pm 6.4)$ when compared with the control group $(60.2 \pm 4.0, p<0.001)$. Treatment of diabetic animals with ASTX increased normal morphology and viable spermatozoa compared to the STZ-induced group (normal sperm morphology, $p<0.001$; viability, $p=0.03$ ) (Table 1 ).

\section{Sperm nuclear chromatin \\ 1) $A O$ staining}

AO determines the susceptibility of sperm nuclear DNA to in situ acid-induced denaturation by the shift of $\mathrm{AO}$ fluorescence from green (nondenatured DNA) to orange-red (denatured DNA) (Figure 1).

Figure 2 indicates that the mean percentage of red spermatozoa was increased in STZ-induced rats $(29.5 \pm 4.2)$ compared to the control group $(12.3 \pm 0.57, p<0.001)$.

Treatment with ASTX significantly decreased the mean percentage of AO-positive spermatozoa in the STZ+ASTX group (21.6 \pm 2.8$)$ in comparison with the STZ-induced rats $(29.5 \pm 4.2, p=0.03)$ (Figure 2).

\section{2) CMA3 staining}

CMA3 binds to DNA in protamine-deficient spermatozoa and sperm appears bright yellow (CMA3-positive), while in normal spermatozoa, CMA3 cannot bind to DNA and therefore spermatozoa appear dull

Table 1. Sperm parameters

\begin{tabular}{lccc}
\hline Parameter & Control & STZ & STZ+astaxanthin \\
\hline Total sperm count (per milliliter) & $148.6 \pm 12.3$ & $\left.108.7 \pm 4.8^{\mathrm{a}}\right)$ & $123.0 \pm 10.3^{\mathrm{b})}$ \\
Normal morphology (\%) & $67.4 \pm 3.1$ & $60.7 \pm 1.7$ & $80.6 \pm 8.5^{\mathrm{b}, \mathrm{c}}$ \\
Viability (\%) & $60.2 \pm 4.0$ & $17.5 \pm 6.4^{\mathrm{a}}$ & $\left.26.7 \pm 2.4^{\mathrm{a}, \mathrm{d}}\right)$ \\
Motility (\%) & $57.6 \pm 5.6$ & $43.5 \pm 5.3^{\mathrm{b})}$ & $35.7 \pm 2.9^{\mathrm{a})}$ \\
\hline
\end{tabular}

Values are presented as mean \pm standard deviation.

STZ, streptozotocin; STZ+ASTX, streptozotocin+astaxanthin.

a) $p<0.001,{ }^{\text {b) }} p<0.01$ vs. Control group; ${ }^{\text {cl }} p=0.001$, ${ }^{\text {d) }} p<0.05$, vs. STZ group. 

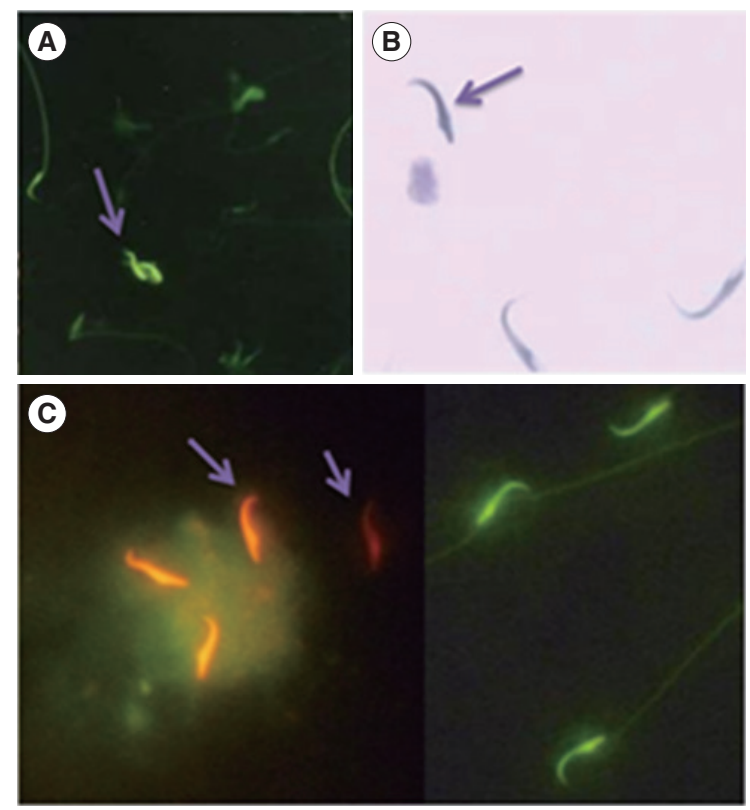

Figure 1. Evaluation of the sperm nuclear chromatin integrity. (A) Chromomycin A3 (CMA3), bright yellow stained spermatozoa were considered as CMA3 positive or protamine deficient (arrow) while green stained spermatozoa were considered to be CMA3 negative, with a normal amount of protamine. (B) Toluidine blue staining, dark blue stained (arrow) were abnormal spermatozoa and unstained or pale blue stained were normal spermatozoa. (C) Acridine orange (AO), orange-red stained (arrows) were abnormal spermatozoa (denatured DNA) while green stained spermatozoa were considered to be AO negative (non-denatured DNA) $(\times 1,000)$.

yellow (CMA3-negative) (Figure 1).

Figure 2 represents the percentage of CMA3-positive spermatozoa in the experimental groups.

The mean percentages of CMA3-positive spermatozoa were $4.6 \%$ $\pm 2.7 \%, 9.7 \% \pm 1.7 \%$, and $7 \% \pm 1.8 \%$ in the control, STZ, and STZ +ASTX groups, respectively. A declining trend was seen in the STZ +ASTX group compared to the STZ group, but it was not significant.

\section{3) TB staining}

TB measures the rate of sperm nuclear chromatin condensation by binding to the phosphate groups of DNA strands (Figure 1). As shown in Figure 2, the percentage of abnormal chromatins in TB staining increased in the STZ group $(38.7 \% \pm 7.5 \%)$ compared to the control group (10.6 $\pm 5.6, p<0.001)$. ASTX attenuated these abnormal chromatins compared with the STZ group $(p=0.001)$ (Figure 2).

\section{Discussion}

We have embarked on this study to determine the detrimental effect that the progression of DM has on sperm parameters and DNA

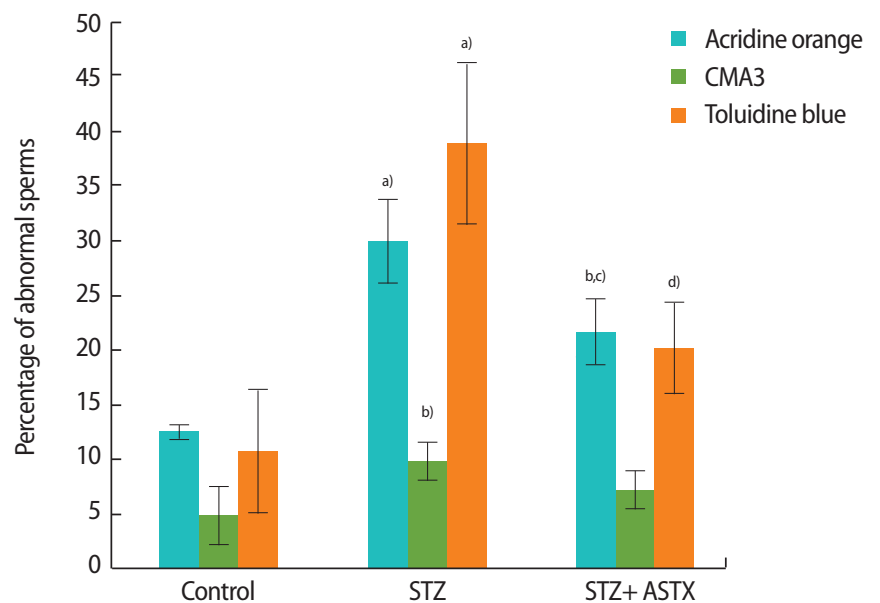

Figure 2. The percentage of abnormal sperm chromatin. Results of chromomycin A3 (CMA3), toluidine blue, and acridine orange staining in control, spermatozoa function in streptozotocin (STZ) and STZ+ astaxanthin (ASTX) groups. a) $p<0.001$ vs. control group; ${ }^{\mathrm{b}} p<0.05$ vs. control group; $;^{\text {c }} p<0.05$ vs. STZ group; ${ }^{d)} p=0.001$ vs. STZ group.

integrity, and whether ASTX could attenuate the complications of diabetes. This investigation indicated that ASTX-treated diabetic rats have significantly higher normal sperm morphology and viability. Analyses of sperm chromatin integrity by $\mathrm{AO}$ and $\mathrm{TB}$ staining illustrated that ASTX could improve sperm DNA integrity.

An increasing experimental and clinical body of evidence indicates that diabetes and high plasma glucose levels pose a threat for spermatozoa function and can increase the risk of infertility [36,37]. DM might pose problems for the male reproductive system as a result of its effect on the endocrine control of spermatogenesis, steroidogenesis, sperm maturation, and impairment of penile erection [38].

Hyperglycemia results in the increased production of free radicaltriggered oxidative stress and consequently alters sperm parameters and DNA integrity [39]. ROS can initiate DNA fragmentation during the early stage of DM [21]. Many antioxidant agents and Chinese medicinal herbs have been investigated for their role in diminishing ROS activity in the cauda epididymis of male animals, but an exact remedy has not yet been identified [40].

Antioxidant molecules, especially carotenoids, play an important role in the control of the oxidative process. Carotenoids are strong antioxidants due to their double-bonded structure, allowing the delocalization of impaired electrons [41]. ASTX, a photo-protective red pigment belonging to the carotenoid family, is recognized as having anti-oxidant, anti-cancer, anti-diabetic, and anti-inflammatory properties and is present in many dietary supplements. ASTX antioxidant capabilities are about 10 times greater than that of other carotenoids and 100 times greater than that of a-tocopherol [19,42].

Considerable evidence points to the defensive role of ASTX against 
DM. The anti-apoptotic and anti-inflammatory role of ASTX in liver, neuronal, and epithelial cells in diabetic animals has been reported $[43,44]$.

Under stressful conditions such as diabetes, normal sperm morphology and viability is reduced $[3,10]$ and ASTX treatment could enhance normal sperm morphology and viability [45]. However, our data showed that ASTX did not improve sperm count or motility in diabetic rats. The observations of the present study might indicate that the extent of oxidative stress has a negative effect on the treatment function of ASTX. Another explanation could be based on the dose-dependent role of ASTX on sperm function, or on how this might relate to oxidative stress, strictly defined. Dona et al. [46] found that ASTX has a dose-response curve for human sperm and an elevated dose of improved sperm parameters such as the acrosome reaction. We intend to consider other important factors such as time of treatment and pre- and post-treatment protocols (i.e., which pretreatment is more effective) in more detail in future work.

However, though many reports have established ASTX as a pretreatment supplement $[47,48]$, our investigation introduced ASTX as a treatment itself; the appropriate duration and dosage of ASTX remain to be determined.

Aitken et al. [49] reported that induction of ROS contributes to DNA fragmentation in sperm nuclei. It has been widely accepted that DM inevitably damages the sperm DNA integrity [50]. The present study specified that ASTX could reduce sperm DNA damage, which was detected by AO or TB staining in STZ-induced rats. On the other hand, ASTX treatment did not show significant differences among the experimental groups with CMA3 staining. Instead, the distinctions among the experimental groups was the same as with $\mathrm{AO}$ and TB staining. During spermiogenesis, histones are replaced by protamines. CMA3 staining was used to determine protamine deficiency [51]. As a result, this staining indicated that DM has no effect on DNA protamines. It seems that other antioxidants such as vitamins and herbal supplements have no effect on histone-protamine replacement [51,52], and ASTX also failed to improve the level of protamines in comparison to the STZ or control groups.

In conclusion, taken together, the current study showed that in vivo ASTX treatment could partially improve sperm viability, normal morphology, and DNA integrity. However, there are some unresolved problems concerning the precise effect of ASTX on sperm function, for which more clarification is needed through future studies. Therefore, further studies should assess testes and epididymis tissues, and determine oxidative stress markers such as lipid peroxidation, advanced protein oxidation products, nitric oxide, glutathione, or the activity of superoxide dismutase and catalase in the sperm and sex organ tissues.

\section{Conflict of interest}

No potential conflict of interest relevant to this article was reported.

\section{References}

1. Ugarte M, Brown M, Hollywood KA, Cooper GJ, Bishop PN, Dunn WB. Metabolomic analysis of rat serum in streptozotocin-induced diabetes and after treatment with oral triethylenetetramine (TETA). Genome Med 2012;4:35.

2. Wild S, Roglic G, Green A, Sicree R, King H. Global prevalence of diabetes: estimates for the year 2000 and projections for 2030. Diabetes Care 2004;27:1047-53.

3. La Vignera S, Condorelli R, Vicari E, D’Agata R, Calogero AE. Diabetes mellitus and sperm parameters. J Androl 2012;33:145-53.

4. Mallidis C, Czerwiec A, Filippi S, O’Neill J, Maggi M, McClure N. Spermatogenic and sperm quality differences in an experimental model of metabolic syndrome and hypogonadal hypogonadism. Reproduction 2011;142:63-71.

5. Sexton WJ, Jarow JP. Effect of diabetes mellitus upon male reproductive function. Urology 1997;49:508-13.

6. Johansen JS, Harris AK, Rychly DJ, Ergul A. Oxidative stress and the use of antioxidants in diabetes: linking basic science to clinical practice. Cardiovasc Diabetol 2005;4:5.

7. Agarwal A. Role of oxidative stress in male infertility and antioxidant supplementation [Internet]. Cleveland: American Center for Reproductive Medicine; 2005 [cited 2016 May 16]. Available from: https://www.clevelandclinic.org/reproductiveresearchcenter/docs/agradoc174.pdf.

8. Evans MD, Dizdaroglu M, Cooke MS. Oxidative DNA damage and disease: induction, repair and significance. Mutat Res 2004; 567:1-61.

9. Agbaje IM, Rogers DA, McVicar CM, McClure N, Atkinson AB, Mallidis $C$, et al. Insulin dependant diabetes mellitus: implications for male reproductive function. Hum Reprod 2007;22: 1871-7.

10. Mangoli E, Talebi AR, Anvari M, Pourentezari M. Effects of experimentally-induced diabetes on sperm parameters and chromatin quality in mice. Iran J Reprod Med 2013;11:53-60.

11. Aitken RJ, Baker MA. Oxidative stress, sperm survival and fertility control. Mol Cell Endocrinol 2006;250:66-9.

12. Lewis SE. Is sperm evaluation useful in predicting human fertility? Reproduction 2007;134:31-40.

13. Sadi G, Eryilmaz N, Tutuncuoglu E, Cingir S, Guray T. Changes in expression profiles of antioxidant enzymes in diabetic rat kidneys. Diabetes Metab Res Rev 2012;28:228-35.

14. Coppey LJ, Gellett JS, Davidson EP, Dunlap JA, Lund DD, Yorek 
MA. Effect of antioxidant treatment of streptozotocin-induced diabetic rats on endoneurial blood flow, motor nerve conduction velocity, and vascular reactivity of epineurial arterioles of the sciatic nerve. Diabetes 2001;50:1927-37.

15. Sadi G, Yilmaz O, Guray T. Effect of vitamin C and lipoic acid on streptozotocin-induced diabetes gene expression: mRNA and protein expressions of Cu-Zn SOD and catalase. Mol Cell Biochem 2008;309:109-16.

16. Hughes CM, Lewis SE, McKelvey-Martin VJ, Thompson W. The effects of antioxidant supplementation during Percoll preparation on human sperm DNA integrity. Hum Reprod 1998;13:1240-7.

17. Comhaire FH, El Garem Y, Mahmoud A, Eertmans F, Schoonjans F. Combined conventional/antioxidant "Astaxanthin" treatment for male infertility: a double blind, randomized trial. Asian J Androl 2005;7:257-62.

18. Ikeuchi M, Koyama T, Takahashi J, Yazawa K. Effects of astaxanthin in obese mice fed a high-fat diet. Biosci Biotechnol Biochem 2007;71:893-9.

19. Naguib YM. Antioxidant activities of astaxanthin and related carotenoids. J Agric Food Chem 2000;48:1150-4.

20. Naito Y, Uchiyama K, Aoi W, Hasegawa G, Nakamura N, Yoshida N, et al. Prevention of diabetic nephropathy by treatment with astaxanthin in diabetic db/db mice. Biofactors 2004;20:49-59.

21. Shrilatha B, Muralidhara. Early oxidative stress in testis and epididymal sperm in streptozotocin-induced diabetic mice: its progression and genotoxic consequences. Reprod Toxicol 2007;23: 578-87.

22. Abeeleh MA, Ismail ZB, Alzaben KR, Abu-Halaweh SA, Al-Essa MK, Abuabeeleh J, et al. Induction of diabetes mellitus in rats using intraperitoneal streptozotocin: a comparison between 2 strains of rats. Eur J Sci Res 2009;32:398-402.

23. Soudamani S, Yuvaraj S, Malini T, Balasubramanian K. Experimental diabetes has adverse effects on the differentiation of ventral prostate during sexual maturation of rats. Anat $\operatorname{Rec} A$ Discov Mol Cell Evol Biol 2005;287:1281-9.

24. Yuan JP, Peng J, Yin K, Wang JH. Potential health-promoting effects of astaxanthin: a high-value carotenoid mostly from microalgae. Mol Nutr Food Res 2011;55:150-65.

25. Hussein G, Sankawa U, Goto H, Matsumoto K, Watanabe H. Astaxanthin, a carotenoid with potential in human health and nutrition. J Nat Prod 2006;69:443-9.

26. Rosiepen G, Weinbauer GF, Schlatt S, Behre HM, Nieschlag E. Duration of the cycle of the seminiferous epithelium, estimated by the 5-bromodeoxyuridine technique, in laboratory and feral rats. J Reprod Fertil 1994;100:299-306.

27. Yama OE, Duru FI. Temporal adaptation in the testes of rat administered single dose Momordica charantia for three interrupt- ed spermatogenic cycles: cytometric quantification. Middle East Fertil Soc J 2011;16:194-9.

28. Frode TS, Medeiros YS. Animal models to test drugs with potential antidiabetic activity. J Ethnopharmacol 2008;115:173-83.

29. Bahmanzadeh M, Abolhassani F, Amidi F, Ejtemaiemehr Sh, Salehi $M$, Abbasi $M$. The effects of nitric oxide synthase inhibitor (L-NAME) on epididymal sperm count, motility, and morphology in varicocelized rat. Daru 2008;16:23-8.

30. Shokri S, Hemadi M, Bayat G, Bahmanzadeh M, Jafari-Anarkooli I, Mashkani B. Combination of running exercise and high dose of anabolic androgenic steroid, nandrolone decanoate, increases protamine deficiency and DNA damage in rat spermatozoa. Andrologia 2014;46:184-90.

31. Seed J, Chapin RE, Clegg ED, Dostal LA, Foote RH, Hurtt ME, et al. Methods for assessing sperm motility, morphology, and counts in the rat, rabbit, and dog: a consensus report. ILSI Risk Science Institute Expert Working Group on Sperm Evaluation. Reprod Toxicol 1996;10:237-44.

32. Nasr-Esfahani MH, Razavi S, Mardani M. Relation between different human sperm nuclear maturity tests and in vitro fertilization. J Assist Reprod Genet 2001;18:219-25.

33. Erenpreiss J, Jepson K, Giwercman A, Tsarev I, Erenpreisa J, Spano M. Toluidine blue cytometry test for sperm DNA conformation: comparison with the flow cytometric sperm chromatin structure and TUNEL assays. Hum Reprod 2004;19:2277-82.

34. Sadeghi MR, Lakpour N, Heidari-Vala H, Hodjat M, Amirjannati N, Hossaini Jadda $\mathrm{H}$, et al. Relationship between sperm chromatin status and ICSI outcome in men with obstructive azoospermia and unexplained infertile normozoospermia. Rom J Morphol Embryol 2011;52:645-51.

35. Jee BC, Suh CS, Shin MS, Lee HJ, Lee JH, Kim SH. Sperm nuclear DNA fragmentation and chromatin structure in one-day-old ejaculated sperm. Clin Exp Reprod Med 2011;38:82-6.

36. La Vignera S, Vicari E, Condorelli R, D'Agata R, Calogero AE. Ultrasound characterization of the seminal vesicles in infertile patients with type 2 diabetes mellitus. Eur J Radiol 2011;80:e64-7.

37. Ding GL, Liu Y, Liu ME, Pan JX, Guo MX, Sheng JZ, et al. The effects of diabetes on male fertility and epigenetic regulation during spermatogenesis. Asian J Androl 2015;17:948-53.

38. Jangir RN, Jain GC. Diabetes mellitus induced impairment of male reproductive functions: a review. Curr Diabetes Rev 2014; 10:147-57.

39. Karimi J, Goodarzi MT, Tavilani H, Khodadadi I, Amiri I. Increased receptor for advanced glycation end products in spermatozoa of diabetic men and its association with sperm nuclear DNA fragmentation. Andrologia 2012;44 Suppl 1:280-6.

40. Hisatomi A, Sakuma S, Fujiwara M, Seki J. Effect of tacrolimus on 
the cauda epididymis in rats: analysis of epididymal biochemical markers or antioxidant defense enzymes. Toxicology 2008;243: 23-30.

41. El-Agamey A, Lowe GM, McGarvey DJ, Mortensen A, Phillip DM, Truscott TG, et al. Carotenoid radical chemistry and antioxidant/ pro-oxidant properties. Arch Biochem Biophys 2004;430:37-48.

42. Kim JH, Nam SW, Kim BW, Kim WJ, Choi YH. Astaxanthin improves the proliferative capacity as well as the osteogenic and adipogenic differentiation potential in neural stem cells. Food Chem Toxicol 2010;48:1741-5.

43. Sila A, Kamoun Z, Ghlissi Z, Makni M, Nasri M, Sahnoun Z, et al. Ability of natural astaxanthin from shrimp by-products to attenuate liver oxidative stress in diabetic rats. Pharmacol Rep 2015; 67:310-6.

44. Dong LY, Jin J, Lu G, Kang XL. Astaxanthin attenuates the apoptosis of retinal ganglion cells in $\mathrm{db} / \mathrm{db}$ mice by inhibition of oxidative stress. Mar Drugs 2013;11:960-74.

45. Tripathi DN, Jena GB. Astaxanthin inhibits cytotoxic and genotoxic effects of cyclophosphamide in mice germ cells. Toxicology 2008;248:96-103.

46. Dona G, Kozuh I, Brunati AM, Andrisani A, Ambrosini G, Bonanni $\mathrm{G}$, et al. Effect of astaxanthin on human sperm capacitation. Mar Drugs 2013;11:1909-19.

47. Mansour N, McNiven MA, Richardson GF. The effect of dietary supplementation with blueberry, alpha-tocopherol or astaxanthin on oxidative stability of Arctic char (Salvelinus alpinus) semen. Theriogenology 2006;66:373-82.

48. Park CH, Xu FH, Roh SS, Song YO, Uebaba K, Noh JS, et al. Astaxanthin and Corni Fructus protect against diabetes-induced oxidative stress, inflammation, and advanced glycation end product in livers of streptozotocin-induced diabetic rats. J Med Food 2015;18:337-44.

49. Aitken RJ, Gordon E, Harkiss D, Twigg JP, Milne P, Jennings Z, et al. Relative impact of oxidative stress on the functional competence and genomic integrity of human spermatozoa. Biol Reprod 1998;59:1037-46.

50. Alves MG, Martins AD, Rato L, Moreira PI, Socorro S, Oliveira PF. Molecular mechanisms beyond glucose transport in diabetesrelated male infertility. Biochim Biophys Acta 2013;1832:626-35.

51. Talebi AR, Mangoli E, Nahangi $H$, Anvari M, Pourentezari $M$, Halvaei I. Vitamin C attenuates detrimental effects of diabetes mellitus on sperm parameters, chromatin quality and rate of apoptosis in mice. Eur J Obstet Gynecol Reprod Biol 2014;181: 32-6.

52. Ghosh A, Jana K, Pakhira BP, Ghosh D. Antiapoptotic efficacy of seed of Eugenia jambolana on testicular germ cell in experimental diabetic rat: a genomic study. Andrologia 2016;48:282-92. 